\title{
CONSERVATIVE TREATMENT PROTOCOL FOR PARA-KERATINIZED ODONTOGENIC KERATOCYSTS BY ENUCLEATION AND OPEN PACKING
}

\author{
Essam M.Ashour* and Nermine Ramadan ${ }^{* *}$
}

\begin{abstract}
Background: odontogenic keratocyst is classified as a developmental cyst derived from enamel organ or from dental lamina. Treatment of odontogenic keratocysts of the jaws remains controversial.
\end{abstract}

Aim of study: was to report the outcome of a conservative treatment protocol in the form of enucleation with open packing for surgical treatment of parakeratinized odontogenic keratocysts.

Methods: after clinical and radiographic examination, fine needle aspiration was obtained from lesions and submitted for histopathological examination. Then the patients were treated by enucleation and open packing. This conservative treatment protocol was selected to decrease the morbidity rate in young aged patients. The follow-up duration of the cases was 2 years. All the cases were monitored continuously with clinical evaluations and radiographically through Orthopantomographs (OPG) and Cone beam computed tomography (CBCT).

Results: Clinical evaluation showed healing improvement of the surgical site with no signs of infection. Radiographic examination showed newly formed bony trabeculation at surgical site.

Conclusions: This conservative treatment protocol for odontogenic keratocysts based on enucleation with open packing would be a possible choice with a view of offering low recurrence rate and low morbidity rate particularly in young patients.

KEYWORDS: keratocysts; odontogenic tumors; jaw cysts; marsupialization, enucleation, open packing.

* Assistant Professor of Oral and Maxillofacial Surgery, Faculty of Dentistry, October 6 University. Head of Oral and Maxillofacial Surgery Unit, October 6 University Hospital.

** Lecturer of Oral and Maxillofacial Surgery, Faculty of Dentistry, October 6 University. 


\section{INTRODUCTION}

Odontogenic Keratocyst (OKC) is classified as a developmental cyst derived from enamel organ or from dental lamina. It was first identified and described in $1876 .^{(1-3)}$

"Odontogenic Keratocyst" was first proposed by Philipsen in 1956, when he separated seven jaw cysts from cholesteatomas occurring in other cranial areas. In 1962, Pindborg and Hansen suggested the histologic criteria necessary to diagnose OKC. ${ }^{(4)}$

OKCs had been redesigned and classified as Keratocystic Odontogenic Tumors (KCOTs) and defined by the World Health Organization in 2005 as a benign uni-cystic or multi-cystic, intra-osseous tumor of odontogenic origin, with a characteristic lining of para-keratinized stratified squamous epithelium and a potential for aggressive, infiltrative behaviour, and high recurrence rate. ${ }^{(5,6)}$

Again, WHO had re-categorized (KCOTs) into Odontogenic and non-Odontogenic developmental cysts in 2017. It is no longer considered a neoplasm as the evidence supporting that hypothesis like clonality is considered insufficient. ${ }^{(7)}$

The Journey of classifications and nomenclature of OKC is as follows: ${ }^{(8)}$

\begin{tabular}{|l|l|}
\hline Dental cyst & John Hunter 1774 \\
\hline Dermoid Cyst & Mikulicz 1876 \\
\hline Primordial cyst & Robinson 1945 \\
\hline Odontogenic keratocyst & $\begin{array}{l}\text { Philisen 1956 \& } \\
\text { Pindborg and Hansen 1963 }\end{array}$ \\
\hline Benign neoplasm & Toller 1967 \\
\hline Odontogenic keratocyst & WHO 1971 \\
\hline $\begin{array}{l}\text { True benign cystic } \\
\text { epithelial neoplasm }\end{array}$ & Ahlfors 1984 \\
\hline Odontogenic keratocyst & WHO 1992 \\
\hline Keratocystoma & Shear 2003 \\
\hline $\begin{array}{l}\text { keratocystic odontogenic } \\
\text { tumor (Benign neoplasm) }\end{array}$ & WHO 2005 \\
\hline Odontogenic keratocyst & WHO 2017 \\
\hline
\end{tabular}

OKCs comprise approximately $11 \%$ of all cysts of the jaws. Clinically, patients may present with swelling, pain and discharge or may be asymptomatic. ${ }^{(9)}$

OKC shows a bimodal age distribution with its first peak in second and third decade and the second peak in fifth and sixth decade of life. It is more commonly seen in males with a M:F ratio of $1.3: 1^{(10)}$ and has an increased site predilection for posterior body and ramus of mandible, with the maxillary involvement being very rare $(<1 \%) \cdot{ }^{(11)}$ Diagnosis of $\mathrm{OKC}$ is confirmed by histopathology though radiographic features may be suggestive of it.

Radiographically, it is most often unilocular or multilocular wellcircumscribed radiolucent lesion surrounded by smooth or scalloped margins with sclerotic borders and can occur in dentigerous relationship causing tooth displacement or sometimes even root resorption. In 25-40\% of cases, impacted teeth are present in the lesion. ${ }^{(12)}$ Although OKC tends to grow in medullary spaces without significant bony expansion, it can cause extensive bone destruction. ${ }^{(13)}$

CT scanning may be helpful in detecting cortical perforation and assessment of soft tissue involvement. Moreover, additional information about lesion can be obtained with the help of computed tomography where the tumor contents usually have low attenuation typical of fluid with low concentrated protein. On magnetic resonance images, OKC shows low to intermediate signal intensity on T1- weighted images and high signal intensity on $\mathrm{T} 2$-weighted images. ${ }^{(14)}$

Microscopically, OKC is characterized by $8-10$ cell layers thick stratified squamous epithelium with hyper-chromatic and palisaded basal cell layer (characteristic of true OKCs) having cuboidal or columnar cells arranged in palisaded fashion described as "picket fence or tombstone appearance" with lack of rete ridges/pegs. The luminal surface has flattened, parakeratotic epithelial cells exhibiting a 
corrugated appearance. ${ }^{(15)}$ The fibrous connective tissue wall contained sparse chronic inflammatory cells which were composed of lymphocytes and plasma cells. Fibrous connective tissue wall may get mineralized and may include cholesterol crystals and Rushton bodies. ${ }^{(16)}$

On a microscopic level, Crowley et al. found two variants of the cyst lining exist: para-keratinized epithelial lining and ortho-keratinized epithelial lining, with a frequency of approximately $86.2 \%$ and $12.2 \%$, respectively. $1.6 \%$ of lesions examined had features of both para-keratinized and orthokeratinized epithelial lining. ${ }^{(17)}$

OOC shows prominent granular layer with orthokeratinized surface and does not show pallisading of basal cells and is histologically different from para-keratinized OKC showing less aggressive behaviour and destruction. The recurrence rate in OOC is very less when compared to OKC and it is not associated with nevoid basal cell carcinoma syndrome (NBCCS). The recurrence rate, aggressive behavior, and neoplastic potential of $\mathrm{OKC}$ are higher, which suggests the importance of distinguishing between $\mathrm{OKC}$ and OOC. ${ }^{(18)}$

Radiographically, OOCs tend to be unilocular lesions and are more often associated with impacted teeth as compared to OKC.

Some studies had reported recurrence rates for intra-osseous odontogenic keratocysts ranging from $5 \%$ to $62 \%$, although several studies examining a large number of cases indicate a recurrence rate of approximately $20-30 \%$ up to 10 years after treatment, though it is more common during the first 5-7 years. ${ }^{(19-21)}$

In 1976, Brannon proposed 3 mechanisms for OKC recurrence: incomplete removal of the cyst lining, growth of a new OKC from satellite cysts/ daughter cysts(or odontogenic rests left behind after surgery) and development of a new OKC in an adjacent area that is interpreted as a recurrence. ${ }^{(22)}$

More recently, Singh et al reported that other factors as thin and friable nature of the epithelial lining, the higher level of cell proliferative activity in the epithelium and budding in the basal layer of the epithelium together with cortical perforation and adherence to adjacent soft tissues are other contributors to the high recurrence rate of $\mathrm{OKC}$ after surgical treatment. ${ }^{(16)}$

OKCs are characterized by their aggressive growth and high propensity to recur following surgical treatment. ${ }^{(23)}$ Most cases recur within the first 5 years after treatment. ${ }^{(20)}$ Recurrence rate varies from 5 to $62 \% .^{(19-21)}$ This much varied recurrence is due to varying periods of follow up, inclusion of orthokeratinized odontogenic cyst or not, the different number of cases incorporated into the studies and also on treatment modality adopted.

Numerous surgical techniques had been advocated in order to facilitate their removal and reduce risk of recurrence. These techniques include enucleation with primary closure, ${ }^{(24)}$ enucleation with excision of the overlying mucosa and open packing, ${ }^{(25)}$ enucleation followed by peripheral ostectomy, ${ }^{(26)}$ enucleation accompanied by chemical fixation of surrounding tissues using Carnoy's solution, ${ }^{(27,28)}$ enucleation accompanied by thermal fixation (cryotherapy) using liquid nitrogen, ${ }^{(29)}$ marsupialization and decompression with subsequent enucleation, ${ }^{(30-33)}$ and marginal or segmental resection of the involved jaw. ${ }^{(34)}$

However, the success of treatment depends mainly upon the involved site, size of lesion, proximity of vital structures, and appropriate surgical procedure with regular clinical follow-up. ${ }^{(20)}$

Treatment of OKCs remains a controversial subject $^{(14)}$. Most cases recur within the first 5 years following the treatment ${ }^{(15,16)}$. Many attempts have been made to reduce the high recurrence rate by improving the operative technique. Advocates of conservative treatments suggest that marsupialization yields results comparable to those obtained with more extensive surgery. ${ }^{(25,28)}$ 
The causes and factors responsible for KCOT recurrence are: ${ }^{(8)}$

1. Incomplete removal of cystic lining

2. Thin and friable nature of epithelial lining,

3. Higher level of cell proliferative activity in the epithelium.

4. Budding in the basal layer of the epithelium

5. Bony perforation.

6. Adherence to adjacent soft tissue.

7. Supraepithelial and Subepithelial split of the epithelial lining.

8. Parakeratinization of the surface layer

9. Remnants of dental lamina epithelium not associated with original $\mathrm{OKC}$ and development of new OKC in the adjacent area.

10. Growth of new OKC from satellite cyst/ daughter cyst/remnants/cell rests.

\begin{tabular}{|l|l|}
\hline enucleation & $30 \%$ \\
\hline enucleation + carnoy' solution & $9 \%$ \\
\hline enucleation + peripheral ostectomy & $18 \%$ \\
\hline $\begin{array}{l}\text { enucleation + carnoy' solution + peripheral } \\
\text { ostectomy }\end{array}$ & $8 \%$ \\
\hline enucleation + cryotherapy & $38 \%$ \\
\hline marsupialization & $33 \%$ \\
\hline marsupialization+ cystectomy & $13 \%$ \\
\hline Resection & $0 \%$ \\
\hline
\end{tabular}

Table showing Recurrence rates with different treatment modalities (Madras and Lapointe, $2008)^{(35)}$

Aim of study: the aim of the current study was to report the outcome of conservative treatment protocol composed of enucleation with open packing for treatment of parakeratinized odontogenic keratocysts.

\section{PATIENTS AND METHODS}

The study was conducted at the Oral and Maxillofacial Surgery Department, Faculty of Dentistry, October 6 University from February 2016 to November 2018 after obtaining permission from the Ethical Committee of O6U.

In this study, 12 patients ranging in age from 9 to 20 years were included and followed for 2 years. The patients were free from any systemic disorders. In clinical examination, mild swelling was noticed on same side of lesion, Figure (1). The overlying mucosa showed slight inflammation. There was no any regional cervical lymphadenopathy.

All patients were evaluated radiographically by Orthopantomographs (OPG) and cone beam computed tomography (CBCT). The imaging revealed a multilocular radiolucency. Figure $(2,3)$

Fine needle aspiration yielded white creamy viscid material which is considered a characteristic of OKC. Tissues obtained from the lesion were submitted for histopathological examination.

Microscopic examination revealed epithelial lining of parakeratinized stratified squamous epithelium. The basal layer of the epithelium was composed of hyalinized fibrous connective tissue.

Histological diagnosis of ortho-keratinized odontogenic cyst was excluded from study because of different biological behaviour.

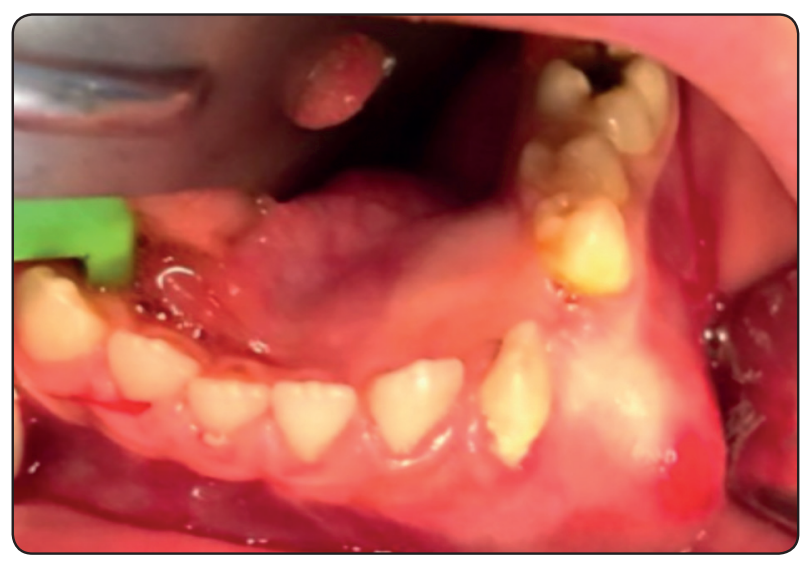

Fig. (1): pre-operative intra-oral clinical photograph showing swelling related to canine premolar region in case No 1 


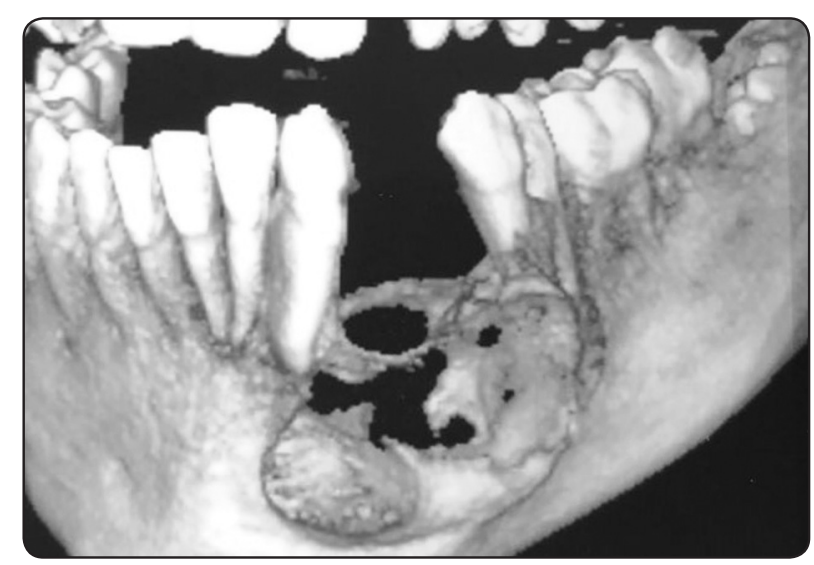

Fig. (2): pre-operative CBCT showing multilocular radiolucent lesion related to mandibular left premolar area in case No 1

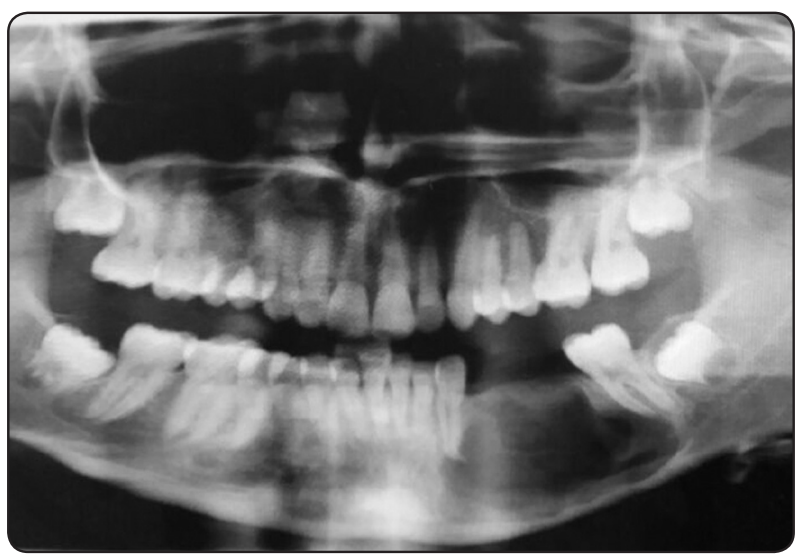

Fig. (3): pre-operative OPG showing unilocular radiolucent lesion related to mandibular left premolar-molar area causing root resorption in case No 2

A conservative treatment protocol was advocated in the form of enucleation with open packing. Under Local anesthesia, an incision was made and a full thickness mucoperiosteal flap was raised. The cyst lining was enucleated and the cystic cavity was curetted removing any pathological lining, the overlying mucosa was excised and the resulting cavity was packed with iodoform gauze impregnated with bacitracin ointment. The surgical cavity was irrigated with sterile saline and the packing was replaced every three days for the first month then weekly for the next 6 months then biweekly throughout the recall visits until healing occurred. Figures $(4,5)$

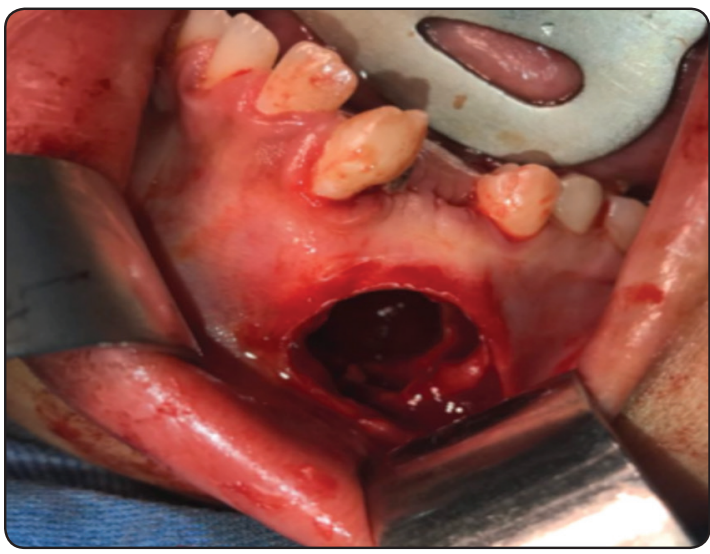

Fig. (4): intra-operative clinical photograph of the 12 years patient showing enucleation of $\mathrm{OKC}$ in case No 1 .

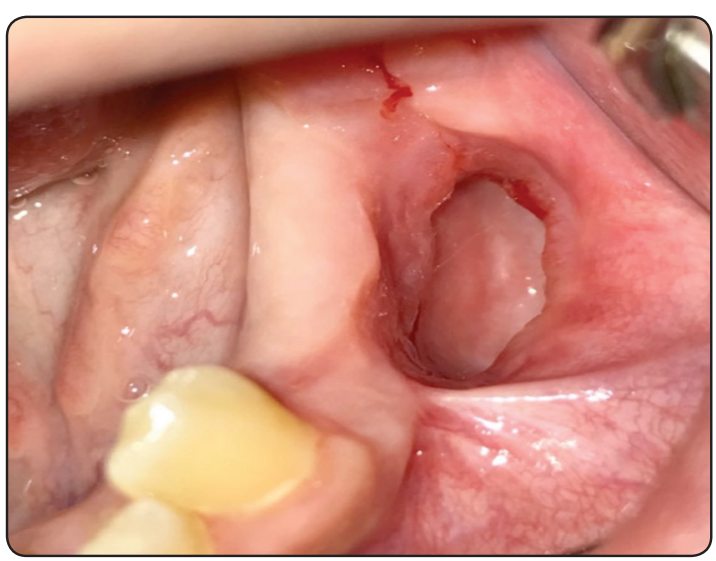

Fig. (5): Postoperative intraoral view of clinical follow up of OKC showing keratin layer formation after 3 months following the surgery in case No 2 .

The patients were reviewed radiologically every three months with Ortho-pantomogram and CBCT during follow-up period. At the end of 2 years follow-up period, no evidence of recurrence was noticed.

\section{RESULTS}

Clinical evaluation: showed healing improvement of the surgical site with no signs of infection. Figure $(5,6)$

Radiographic examination: showed newly formed bony trabeculation at surgical site. Figure $(7,8)$ 


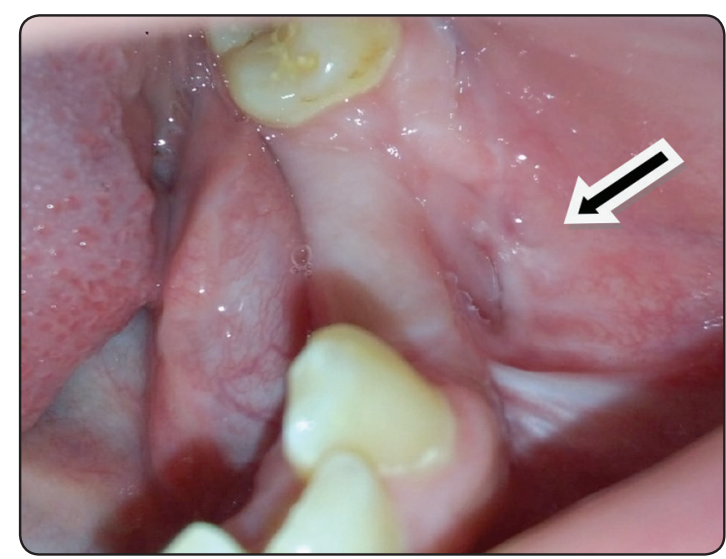

Fig. (6): Postoperative intraoral view of clinical follow up of $\mathrm{OKC}$ showing keratin layer formation after 12 months following the surgery in case No 2 .

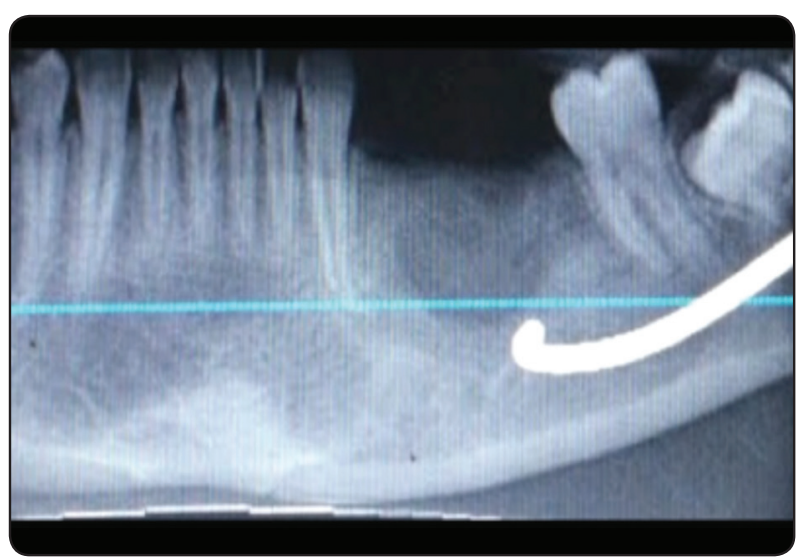

Fig. (7): Post-operative panoramic radiograph showing newly formed bony trabeculation at surgical site.

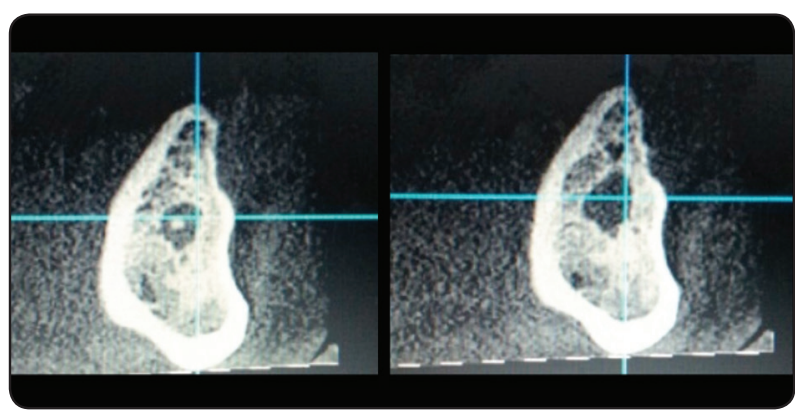

Fig. (8): 2 years post-operative CBCT showing newly formed bony trabeculation at the surgical site.

\section{DISCUSSION}

In a study conducted by Brannon (22), OKCs occurred most frequently in 3rd and 4th decades and Blanas and colleagues ${ }^{(24)}$ demonstrated the peak incidence in 4th and 5th decade. OKCs are rare in extremes of age i.e. under 10 and above 70. Male predilection was noticed with male to female ratio of $1.3: 1$ which also conforms to the previous studies by Blanas and collegues.

Our study was in agreement with other studies $^{(22,24)}$ which announced that OKCs predominantly affect the mandible especially posterior mandible including body, angle and ramus region.

OKCs are characterized by their aggressive growth and high propensity to recur following surgical treatment. ${ }^{(23)}$ Although various treatment modalities for $\mathrm{OKC}$ have been documented in literature, the universally accepted approach remains undecided. These range from conservative methods such as enucleation, marsupialization, curettage, chemical cauterization, peripheral ostectomy to much aggressive treatments such as marginal or segmental resection. ${ }^{(23)}$ Recurrence rate varies from 5 to $62 \% .^{(19-21,35)}$ This much varied recurrence is due to varying periods of follow up, inclusion of orthokeratinized odontogenic cyst or not, the different number of cases incorporated into the studies and also on treatment modality adopted.

Higher recurrence rates have been documented with conservative procedures such as marsupialization and enucleation of the lesion while aggressive therapies, like resection, significantly lowered the recurrence rates and improved prognosis..$^{(18,19,35)}$

Large OKCs are often managed by marsupialization, which relieves the pressure within the cystic cavity and allows new bone to fill the defect with minimal surgical morbidity. Structures such as teeth, the maxillary sinus, or the inferior alveolar canal can be saved from surgical damage. The procedure is highly successful in decreasing the cyst size before enucleation, and is useful to avoid extensive surgery, and is considered the first option 
for the treatment of large OKCs. ${ }^{(18,19)}$ However, this method have high recurrence rate. ${ }^{(35)}$

Unfortunately marsupialization leaves behind pathological tissues increasing the opportunity for the ingrowth of satellite and daughter cysts thus giving rise to a high recurrence rate.

This was evident and documented by Brannon in 1976, who proposed 3 mechanisms for OKC recurrence: incomplete removal of the cyst lining, growth of a new OKC from satellite cysts/daughter cysts(or odontogenic rests left behind after surgery) and development of a new $\mathrm{OKC}$ in an adjacent area that is interpreted as a recurrence. ${ }^{(22)}$

As it is well known that the ultimate goal of surgical procedures is to control and to reduce the potential for recurrence without increasing morbidity to the patients.

Accordingly, in our study, we adopted a conservative treatment protocol by means of enucleation and open packing inorder to eradicate the entire pathological cyst lining thus decreasing the possibility of recurrence meanwhile adopting the desirable benefits of marsupialization by decompression and decreasing the size of the lesion, allowing new bone to fill the defect and decreasing the morbidity by avoiding surgical damage to vital structures and at the same time avoiding patient disfigurement and disability as a side effect of the aggressive radical resection modality.

Our study was in coincidence with other authors ${ }^{(36-38)}$ who suggested that Enucleation of the OKCs and open packing has been used as another conservative method of surgical treatment with low surgical morbidity and recurrence rate. Regular recall visits are required to ensure cyst involution and the opportunity for appropriate treatment should be an evidence of absence of recurrence.

Morais et al ${ }^{(39)}$ demonstrated that the use of marsupialization followed by enucleation and/ or curettage has been showed optimal results and low recurrence rate, which are in agreement with the results of the present study by using enucleation and open packing. Moreover, this approach is con- sidered an effective and less invasive technique in the treatment of KCOTs, reducing the lesion size by drainage and decompression up to $47 \%$ of the initial size, ${ }^{(39)}$ decreasing the aesthetic and functional damages and allowing the preservation of important anatomical structures. ${ }^{(40)}$

Different views on the recommended duration of radiologic and clinical follow-up are reported in the literature.

According to Forssellet al ${ }^{(36)}$, a 4 year period will suffice for the detection of most recurrences. As it was stated by other authors, the recurrence rate after the treatment of OKCs is very broad and treatment dependent. In our study, at the end of 2 year follow-up period, no evidence of recurrence was registered.

Blanas et al. ${ }^{(24)}$ conducted a systematic review of literature and evaluated the collective recurrence rates reported with various types of the treatment. Forty-five cases were treated by marsupialization with a recurrence rate of $24.4 \%$. Enucleation alone was associated with a $28.7 \%$ recurrence ${ }^{(41)}$.

However, our study was disagreed with Browne ${ }^{(42)}$ who has used three different methods of treatment; marsupialization, enucleation and primary closure, and enucleation with packing-open. He found approximately equal rates of recurrence with the three methods of treatment. This is in coincidence with Kolokythas et al. ${ }^{\left({ }^{30)}\right.}$ The aggressive nature of some OKCs necessitates equally aggressive treatment, whereas long-term follow-up even for non-syndromic patients with the single lesion is of paramount importance. Age of the patient and the site and histological characteristics of the treated lesions are not significantly associated with the incidence of recurrence.

Extraction of teeth affected by the lesion, as well as a generous removal of partially eroded bone and overlying soft tissues, may contribute to lower recurrence rates. Bataineh and Al Qudah ${ }^{(43)}$ advocated that resection without continuity defects as a radical treatment, in which the removal of cyst, teeth, and overlying soft tissue is followed by 
packing of the resulting cavity to minimize the risk of recurrence. In order to minimize the recurrence, eliminating the retention areas around the periapical portion of extracted teeth and the eradication of all pathological cyst lining is of prime importance.

The benefits of this conservative treatment protocol is that it offers minimal surgical morbidity with well accepted patient tolerance, with low recurrence rate together with protection of associated vital structures as the inferior alveolar nerve and developing teeth were less vulnerable to damage.

\section{CONCLUSION}

Odontogenic keratocyst is considered as an aggressive cyst and is associated with high recurrence rate. Treatment of OKCs of the Jaw still remains a controversial thus the choice of one best treatment modality is debatable. Single appropriate treatment is yet to be decided. Surgeons should decide the treatment plan according to various factors such as size and behaviour of the lesion, patient's age, and relation to various anatomical and vital structures. Although Resection is considered one of the treatment modalities that carries the least risk of recurrence, it is associated with a great patient disability and disfigurement and accordingly should be limited to recurrent and aggressive Lesions. Thus, enucleation and adjunctive peripheral procedures can be considered optimal and the treatment of choice for most of the cases.

\section{REFERENCES}

1. Rud J, Pindborg JJ. Odontogenic keratocysts: a follow-up study of 21cases. J Oral Surg. 1969 May;27(5):323-30.

2. Panders AK, Haddlers HN. Solitary keratocysts of the jaws. J Oral Surg. 1969 Dec;27(12):931-8.

3. Philipsen HP. Om keratocystes (Kolesteratomer) and kaeberne. Tandlaegebladet 1956 ; 60:963-71.

4. Pindborg JJ, Philipsen HP, Henriksen J. Studies on odontogenic cyst epithelium. In: Sognnaes RF, ed. Fundamentals of Keratinization. Washington, DC: American Association of the Advancement of Science 1962;1:151-60.
5. Barnes L, Eveson JW, Reichart P, Sidransky D, editors. Pathology and genetics of head and neck tumours. Lyon: IARC Press; 2005. WHO classification of tumours series.

6. Reichart PA, Philipsen HP, Sciubba JJ. The new classification of Head and Neck Tumours (WHO) any changes? Oral Oncol. 2006 Sep;42(8):757-8.

7. Wright JM, Vered M. Update from the 4th edition of the world healthorganization classification of head and neck tumours: Odontogenic and maxillofacial bone tumors. Head Neck Pathol 2017;11:68-77.

8. Deepak Passi, DeepikaSinghal, Mahinder Singh, Vijay Mishra, Yoshi Panwar and AanchalSahni. Odontogenic keratocyst (OKC)or keratocystic odontogenic tumor (KCOT) journey of OKC from cyst to tumor to cyst again: comprehensive review with recent updates on WHO classification (2017). International Journal of Current Research July 2017,Vol 9, Issue 07, pp 54080-54086,

9. Maurette PE, Jorge J, de Moraes M. Conservative treatment protocol of odontogenic keratocyst: a preliminary study. J Oral Maxillofac Surg 2006; 64(3):379-83.

10. Shear M. Developmental odontogenic cysts. An update. J Oral Pathol Med 1994; 23:1-11.

11. Ali M, Baughman RA. Maxillary odontogenic keratocyst: A common and serious clinical misdiagnosis. J Am Dent Assoc 2003; 134:877-83.

12. Titinchi F, Nortje CJ. Keratocystic odontogenic tumor: A recurrence analysis of clinical and radiographic parameters. Oral Surg Oral Med Oral Pathol Oral Radiol 2012;114:136-42.

13. Chang NJ, Shyu V, Chen CH. Maxillary reconstruction for a huge odontogenic keratocyst. J CraniofacSurg 2011; 22:1165-67.

14. Yonetsu K, Bianchi JG, Troulis MJ, Curtin HD. Unusual CT Appearance in an Odontogenic Keratocyst of the Mandible: Case Report. Am J Neuroradio 2001 22:1887-89.

15. Arnnop P. Management of odontogenic keratocysts of the jaws. A 10 years experience with 120 consecutive lesions. J CraniomaxillofacSurg 2009; 10: 10-16.

16. Singh HP, Nayar A, Raj A, Kumar P. Are all odontogenic keratocystskeratocystic odontogenic tumors? Correlation between imaging features and epithelial cell proliferation. J Clin Imaging Sci 2013;3:3.

17. Crowley TE, Kaugars GE, Gunsolley JC. Odontogenic keratocysts: A clinical and histologic comparison of the 
parakeratin and orthokeratin variants. J OralMaxillofacSurg 1992;50(1):22-6.

18. Neville BW, Damm DD, Allen CM, Bouquot JE. Oral and maxillofacial pathology 2nd ed. Philadelphia: Saunders; 2002. p. 595.

19. Titinchi F, Nortje CJ. Keratocystic odontogenic tumor: A recurrence analysis of clinical and radiographic parameters. Oral Surg Oral Med Oral Pathol Oral Radiol 2012;114:136-42.

20. Kaczmarzyk T, Mojsa I, Stypulkowska J. A systematic review of therecurrence rate for keratocystic odontogenic tumour in relation to treatment modalities.Int $\mathrm{J}$ Oral MaxillofacSurg 2012;41:756-67.

21. Bhargava D, Deshpande A, Pogrel MA. Keratocystic odontogenic tumour (KCOT) - A cyst to a tumour. Oral MaxillofacSurg 2012;16:163-70.

22.BrannonRB.Theodontogenickeratocyst.Aclinicopathologic study of 312 cases. Part I. Clinical features. Oral Surg Oral Med Oral Pathol 1976; 42(1):54-72.

23. Jurisic M, Andric M, dos Santos JN, Jurisic V (2012). Clinical, diagnostic and therapeutic features of keratocystic odontogenic tumors: a review. J BUON 2012;17: 237-244.

24. Blanas N, Freund B, Schwartz M, Furst IM. Systematic review of the treatment and prognosis of the odontogenic keratocyst. Oral Surg Oral Med Oral Pathol Oral RadiolEndod 2000;90:553-8.

25. Yildirim G, Ataoglu H, Kalayci A, Özkan BT, Kucuk K, Esen A. conservative treatment Protocol for Keratocystic Odontogenic Tumour: a Follow-up Study of 3 Cases. J Oral Maxillofac Res 2010 (Jul-Sep);1(3):e7

26. Pogrel MA. The keratocystic odontogenic tumor. Oral Maxillofac Surg Clin North Am 2013;25:21-30.

27. Güler N, Sençift K, Demirkol O. Conservative management of keratocystic odontogenic tumors of jaws. Scientific World Journal 2012; vol (68) 397.

28. Alchalabi NJ, Merza AM, Issa SA. Using carnoy'solution in treatment of keratocystic odontogenic tumor. Ann MaxillofacSurg 2017;7:51-6.

29. Schmidt BL, Pogrel MA (2001).The use of enucleation and liquid nitrogen cryotherapy in the management of odontogenic keratocysts. Journal of Oral and Maxillofacial Surgery 59: 720-725

30. Kolokythas A, Fernandes RP, Pazok. A. Odontogenic Keratocyst: To decompress or not to decompress? A comparative study of decompression and enucleation versus resection/ peripheral ostectomy. J Oral MaxillofacSurg 2007; 65: 640-44.

31. Ramaswami R, Galinde J, Srivalli N, SidanaS .Keratocystic odontogenic Tumour.Journal of Contemporary Dentistry 2013(2): 87-91

32. Gao L, Wang XL, Li SM, Decompression as a treatment for odontogenic cystic lesions of the jaw. J Oral Maxillofac Surg 2014; 72: 327-333.

33. Smit RB, Moore BK, Lou S MY.Keratocystic Odontogenic Tumour of the Mandible: a case report of decompression with a customised removable tube and review of literature. New Zealand Dental Journal 2015 Sep; 98-101

34. Ghali GE, Connor MS . Surgical management of the odontogenic keratocyst. Oral Maxillofac Surg Clin North Am 2003; 15: 383-392.

35. Madras J \& Lapointe H. Keratocystic odontogenic tumour: reclassification of the odontogenic keratocyst from cyst to tumour. J Can Dental Assoc. 2008, 74(2): 165-165.

36. Forssell K, Forssell H, Kahnberg KE. Recurrence of keratocysts.A long-term follow-up study.Int $\mathrm{J}$ Oral Maxillofac Surg. 1988 Feb;17(1):25-8.

37. Donoff RB, Guralnick WC, Clayman L. Keratocysts of the jaws. J Oral Surg. 1972 Nov;30(11):880-4

38. Gülsün Yildirim, Hanife Ataoglu, Abdullah Kalayci, Birkan Taha Özkan, Korhan Kucuk, Alparslan Esen. Conservative Treatment Protocol for Keratocystic Odontogenic Tumour: a Follow-up Study of 3 Cases. J oral \&maxillof research. Res 2010 (Jul-Sep) | vol. 1;No 3.

39. Morais de Melo W, Pereira-Santos D, Sonoda CK, HochuliVieira E. Decompression for management of keratocystic odontogenic tumor in the mandible. J CraniofacSurg 2012;23: 639-40.

40. Finkelstein MW, Hellstein JW, Lake KS, Vincent SD. Keratocystic odontogenic tumor: A retrospective analysis of genetic, immunohistochemical and therapeutic features. Proposal of a multicenter clinical survey tool. Oral Surg Oral Med Oral Pathol Oral Radiol 2013;116:75-83

41. Jackson IT, Potparic Z, Fasching M, Schievink WI, Tidstrom K, Hussain K. Penetration of the skull base by dissecting keratocyst. J Craniomaxillofac Surg. 1993 Dec;21(8):319-25.

42. Browne RM. The odontogenic keratocyst.Clinical aspects. Br Dent J. 1970 Mar 3;128(5):225-31.

43. Bataineh AB, al Qudah M. Treatment of mandibular odontogenic keratocysts. Oral Surg Oral Med Oral Pathol Oral RadiolEndod. 1998 Jul;86(1):42-7. 